\title{
Laplace Analysis of Periodic Heat and Mass Transport on a Parabolic Started Surface immersed in Darcian Porous Regime
}

\author{
Hamida Khatun*, Sahin Ahmed** \\ *(Department of Mathematics, South Salmara College, Dhubri-783127, Assam, India, \\ Email: azizurdr786@gmail.com) \\ **(Department of Mathematics, Rajiv Gandhi Central University, Rono Hills, Itanagar-791112, \\ Arunachal Pradesh, India, Email: sahin.ahmed@rgu.ac.in)
}

\begin{abstract}
An analysis of periodic heat and mass transport of unsteady hydromagnetic flow past a parabolic started motion of the infinite vertical plate immersed in Darcian porous regime in presence of a first order chemical reaction has been presented. Here the plate temperature as well as concentration level near the plate are increased linearly with time. The boundary layer conservation equations have been solved by Laplace transforms technique. It has been observed that both the velocity and concentration are decreased with increasing values of chemical reaction parameter. But the opposite behavior has been found for the flow velocity when the values of free convection as well as porosity parameter are increased. Application of magnetic fields to medical science is growing rapidly, with the development of novel magnetic pumps, hydromagnetic separation devices with chemical engineering and geophysical energy systems.
\end{abstract}

Keywords: Boundary layer, Chemical engineering, Darcian porous regime, Hydromagnetic, Laplace Transforms Technique.

\section{INTRODUCTION}

Transport processes in porous media can involve fluid, heat and mass transfer in single or multi-phase scenarios. Such flows with and without buoyancy effects arise frequently in many branches of chemical engineering and owing to their viscousdominated nature are generally simulated using the Darcy model. Applications of such flows include chip-based micro fluidic chromatographic separation devices (Dorfman and Brenner, [1]), dissolution of masses buried in a packed bed ( Delgado, [2]), heat transfer in radon saturating permeable regimes (Minkin, [3]), flows in ceramic filter components of integrated gasification combined cycles (IGCC) ( Seo et al., [4]), separation of carbon dioxide from the gas phase with aqueous adsorbents (water and diethanolamine solution) in micro porous hollow fibre membrane modules (Al-Saffar et al., [5]), food processing and polymer production. Keeping in view these applications Chamkha et al. [6] studied the effects of Hydro magnetic combined heat and mass transfer by natural convection from a permeable surface embedded in a fluid saturated porous medium. Mukherjee et al.

[7] studied the momentum and the heat transfer characteristics in incompressible electrically conducting boundary layer flow over an exponentially stretching sheet under the effect of magnetic field with thermal radiation through porous medium. Ahmed [8] investigated the effect of periodic heat transfer on unsteady MHD mixed convection flow past a vertical porous flat plate with constant suction and heat sink when the free stream velocity oscillates in about a non-zero constant mean. There are many applications for the parabolic motion such as solar cookers, solar concentrators and parabolic trough solar collector. A parabolic concentrator type solar cooker has a wide range of applications like baking, roasting and distillation due to its unique property of producing a practically higher temperature of nearly $2500 \mathrm{C}$ and hence it provides inconvenience to the user due to high amount of glare. Solar concentrators have their applications in increasing the rate of evaporation of waste water, in food processing, for making drinking water from brackish and sea water. Many researchers have been attracted due to these important applications namely Kumar et al. [9] investigated about the viscous dissipation effects on the unsteady magnetohydrodynamic free convective flow past a parabolic starting motion of the infinite vertical plate with variable temperature and variable mass diffusion. Muthucumaraswamy and Armstrong [10] presented an exact solution of unsteady flow past a parabolic starting motion of an infinite vertical plate with variable temperature and mass diffusion, in the presence of a homogeneous chemical reaction of first order. Muralidharan and Muthucumaraswamy [11] presented a closed form solution of flow past a parabolic starting motion of the infinite vertical 
plate with uniform heat flux and variable mass diffusion. Muthucumaraswamy and Velmurugan [12] presented the hypothetical solution of flow past a parabolic starting motion of the infinite vertical plate with variable temperature and variable mass diffusion. Muthucumaraswamy and Venkatesan [13] studied an exact solution of unsteady flow past a parabolic started infinite isothermal vertical plate with uniform mass flux, in the presence of thermal radiation. I P a $\mathrm{g}$ e Magnetohydrodynamic flows in porous media have stimulated considerable attention owing to the importance of such flows in magnetic materials processing, chemical engineering and geophysical energy systems. In view of these applications of the flow through porous medium, a series of investigation has been made by Raptis et al. ([14], [15, [16]), where the porous medium is either bounded by horizontal, vertical surfaces or parallel porous plates. Ahmed [17] investigated the unsteady hydromagnetic flow of an electrically conducting fluid through a Darcian porous medium adjacent to a uniformly accelerated vertical plate in a rotating system using boundary layer approximation. Chaudhary and Jain [18] studied the influence of oscillating temperature on magnetohydrodynamic convection heat transfer past a vertical plane in a Darcian porous medium. An investigation was performed for unsteady Magnetohydrodynamic boundary layer flow and heat transfer through a Darcian porous medium bounded by a uniformly moving semi-infinite isothermal vertical plate in presence of thermal radiation by Khatun and Ahmed [19].

Mass diffusion rates can be changed tremendously with chemical reactions. The chemical reaction effects depend whether the reaction is homogeneous or heterogeneous. This depends on whether they occur at an interface or as a single phase volume reaction. In well mixed systems, the reaction is heterogeneous, if it takes place at an interface and homogeneous, if it takes place in solution. In majority cases, a chemical reaction depends on the concentration of the species itself. A reaction is said to be of first order, if the rate of reaction is directly proportional to the concentration of only one reactant and is independent of others. Decomposition of nitrogen pent oxide in the gas phase as well in an organic solvent like $\mathrm{CCl} 4$, conversion of Nchloroacetanilide into p-chloroacetanilide, hydrolysis of methyl acetate and inversion of cane sugar. The radioactive disintegration of unstable nuclei are the best examples of first order reactions. The related literatures are Agrawal et al. [20] studied free convection due to thermal and mass diffusion in laminar flow of an accelerated infinite vertical plate in the presence of magnetic field. Agrawal et al.

[21] further extended the problem of unsteady free convective flow and mass diffusion of an electrically conducting elasto-viscous fluid past a parabolic starting motion of the infinite vertical plate with transverse magnetic plate. The governing equations are tackled using Laplace transform technique. Muthucumaraswamy and Velmurugan [22] studied on the unsteady hydromagnetic flow past a parabolic starting motion of the infinite vertical plate with variable temperature and mass diffusion, in the presence of homogeneous chemical reaction of first order. Manivannan et al. [23] investigated radiation and chemical reaction effects on isothermal vertical oscillating plate with variable mass diffusion. The modal of magnetohydronamic transient convective radiating heat transfers in an isotropic, homogenous porous regime adjacent to a hot vertical plate using the Laplace transform method were studied by Ahmed and Kalita [24]. The effects of conductionradiation, porosity and chemical reaction on unsteady hydromagnetic free convection flow past an impulsively-started semiinfinite vertical plate embedded in a porous medium in the presence of first order chemical reaction and thermal radiation were analyzed by Ahmed [25]. Analytical solution of a magnetohydrodynamic steady mixed convective flow over an infinite vertical porous plate with combined heat and mass transfer is presented by Ahmed et al. [26].

The aim of the present problem is to investigate the effects of porosity of the medium and chemical reaction on the flow past an infinite vertical plate subjected to parabolic motion with variable temperature, in the presence of applied transverse magnetic field. The dimensionless governing equations are solved using the Laplacetransform technique. The solutions are derived in terms of exponential and complementary error functions. The validity of the present flow model has been established through the results compared with the results of the previous work done by Muthucumaraswamy and Velmurugan [22] and the results are in good agreement. 


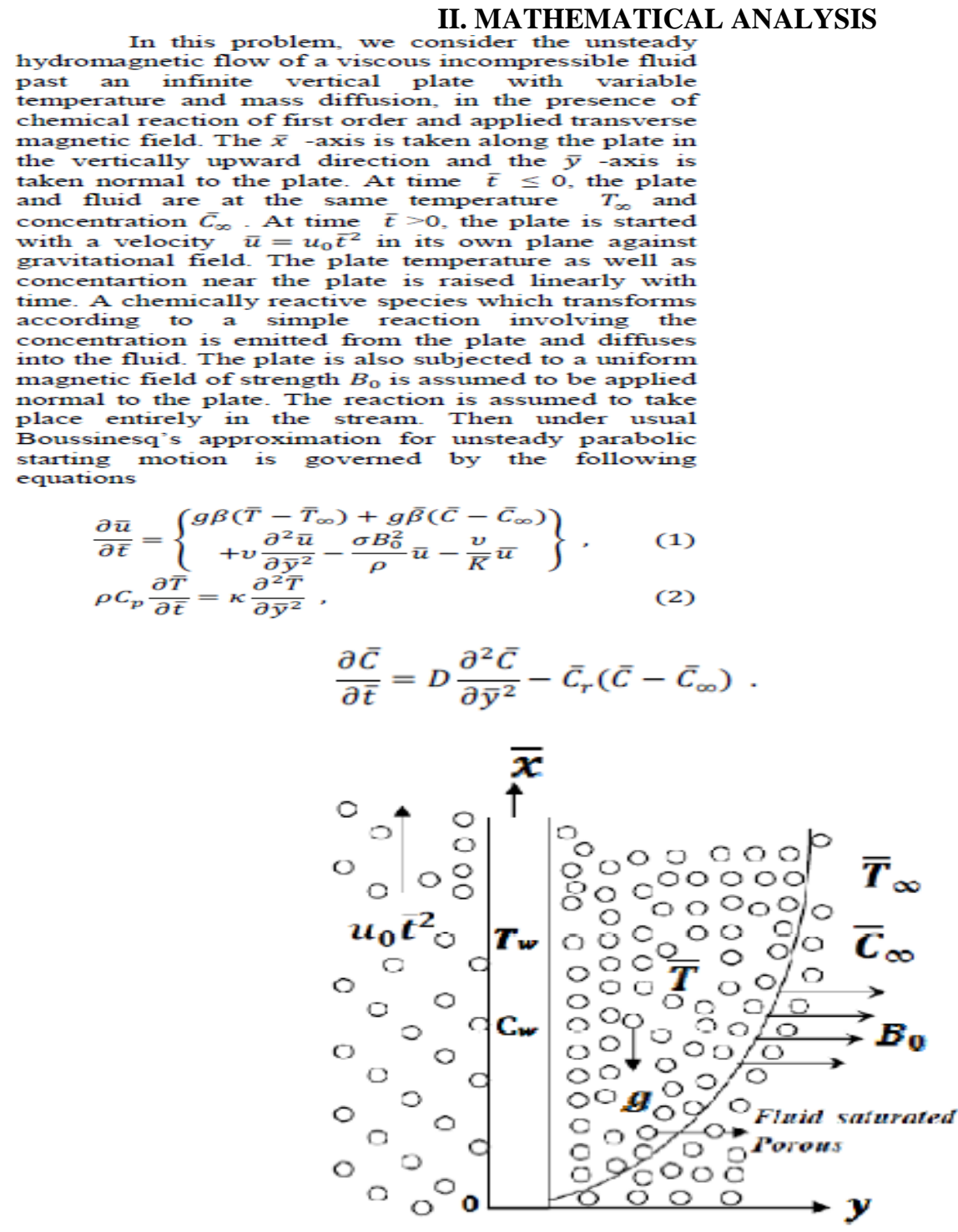

Fig. 1: Physical model and coordinate system

The magnetic and viscous dissipations are neglected in this study. The first and second terms on the RHS of the momentum equation (1) denote the thermal and concentration buoyancy effects, respectively. It is assumed that the permeable plate moves with a variable velocity in the direction of fluid flow. In addition, it is assumed that the temperature and the concentration at the wall are varying with time.

The initial and boundary conditions are

$$
\left.\begin{array}{l}
\left\{\begin{array}{c}
\left(\bar{u}=0, \bar{T}=\bar{T}_{\infty}, \bar{C}=\bar{C}_{\infty}\right) \forall \bar{y}, \bar{t} \leq 0 \\
\bar{t}>0: \\
\bar{u}=u_{0} \bar{t}^{2}, \\
\bar{T}=\bar{T}_{\infty}+\left(\bar{T}_{w}-\bar{T}_{\infty}\right) A \bar{t}, \\
\bar{C}=\bar{C}_{\infty}+\left(\bar{C}_{w}-\bar{C}_{\infty}\right) A \bar{t}
\end{array}\right) \text { at } \bar{y}=0 \\
\bar{t}>0: \\
\left(\begin{array}{c}
\bar{u} \rightarrow 0, \bar{T} \rightarrow \bar{T}_{\infty}, \\
\bar{C} \rightarrow \bar{C}_{\infty}
\end{array}\right) \text { as } \bar{y} \rightarrow \infty
\end{array}\right\},
$$




$$
\left\{\begin{array}{c}
u=\bar{u}\left(\frac{u_{0}}{v^{2}}\right)^{\frac{1}{3}}, t=\bar{t}\left(\frac{u_{0}^{2}}{v}\right)^{\frac{1}{3}}, y=\bar{y}\left(\frac{u_{0}}{v^{2}}\right)^{\frac{1}{3}}, \\
\theta=\frac{T-T_{\infty}}{T_{w}-T_{\infty}}, C=\frac{\bar{C}-\bar{C}_{\infty}}{\bar{C}_{w}-\bar{C}_{\infty}}, \quad G r=\frac{g \beta\left(T_{w}-T_{\infty}\right)}{\left(v u_{0}\right)^{\frac{1}{3}}}, \\
G m=\frac{g \beta\left(\bar{C}_{w}-\bar{C}_{\infty}\right)}{\left(v u_{0}\right)^{\frac{1}{3}}}, M=\frac{\sigma B_{0}^{2}}{\rho}\left(\frac{v}{u_{0}^{2}}\right)^{\frac{1}{3}}, S c=\frac{v}{D}, \\
C_{r}=\bar{C}_{r}\left(\frac{v}{u_{0}^{2}}\right)^{\frac{1}{3}}, \operatorname{Pr}=\frac{\mu C_{p}}{\kappa}, K=\bar{K}\left(\frac{u_{0}}{v^{2}}\right)^{\frac{2}{3}}
\end{array}\right\}
$$

The dimensional equations (1)-(3) reduces to the following non-dimensional form

$$
\begin{aligned}
& \frac{\partial u}{\partial t}=\operatorname{Gr} \theta+G m C+\frac{\partial^{2} u}{\partial y^{2}}-\left(M+K^{-1}\right) u \\
& \frac{\partial \theta}{\partial t}=\frac{1}{\operatorname{Pr}} \frac{\partial^{2} \theta}{\partial y^{2}} \\
& \frac{\partial C}{\partial t}=\frac{1}{S c} \frac{\partial^{2} C}{\partial y^{2}}-C_{r} C
\end{aligned}
$$

The corresponding initial and boundary conditions in non-dimensional form are as follows

$$
\left\{\begin{array}{c}
(u=0, \theta=0, C=0) \forall y, t \leq 0 \\
t>0:\left(u=t^{2}, \theta=t, C=t\right) \text { at } y=0 \\
t>0:(u \rightarrow 0, \theta \rightarrow 0, C \rightarrow 0) \text { as } y \rightarrow \infty
\end{array}\right\} \text { (9) }
$$

\section{METHODOLOGY}

The equations (6)-(8) and the corresponding initial and boundary conditions (9) are solved by the Laplacetransform technique and the solutions are obtained as follows:

$$
\theta=t\left[\begin{array}{c}
\left(1+2 \eta^{2} P r\right) \operatorname{erfc}(\eta \sqrt{P r}) \\
-\frac{2 \eta \sqrt{P r}}{\sqrt{\pi}} \exp \left(-\eta^{2} P r\right)
\end{array}\right]
$$

$$
=\left\{\begin{array}{c}
\frac{t}{2} \exp \left(2 \eta \sqrt{S c C_{r} t}\right) \operatorname{erfc}\left(\eta \sqrt{S c}+\sqrt{C_{r} t}\right) \\
\frac{t}{2} \exp \left(-2 \eta \sqrt{S c C_{r} t}\right) \operatorname{erfc}\left(\eta \sqrt{S c}-\sqrt{C_{r} t}\right) \\
-\frac{\eta \sqrt{S c} \sqrt{t}}{2 \sqrt{C_{r}}} \exp \left(-2 \eta \sqrt{S c C_{r} t}\right) \operatorname{erf} c\left(\eta \sqrt{S c}-\sqrt{C_{r} t}\right) \\
+\frac{\eta \sqrt{S c} \sqrt{t}}{2 \sqrt{C_{r}}} \exp \left(-2 \eta \sqrt{S c C_{r} t}\right) \operatorname{erf} c\left(\eta \sqrt{S c}+\sqrt{C_{r} t}\right)
\end{array}\right\}
$$




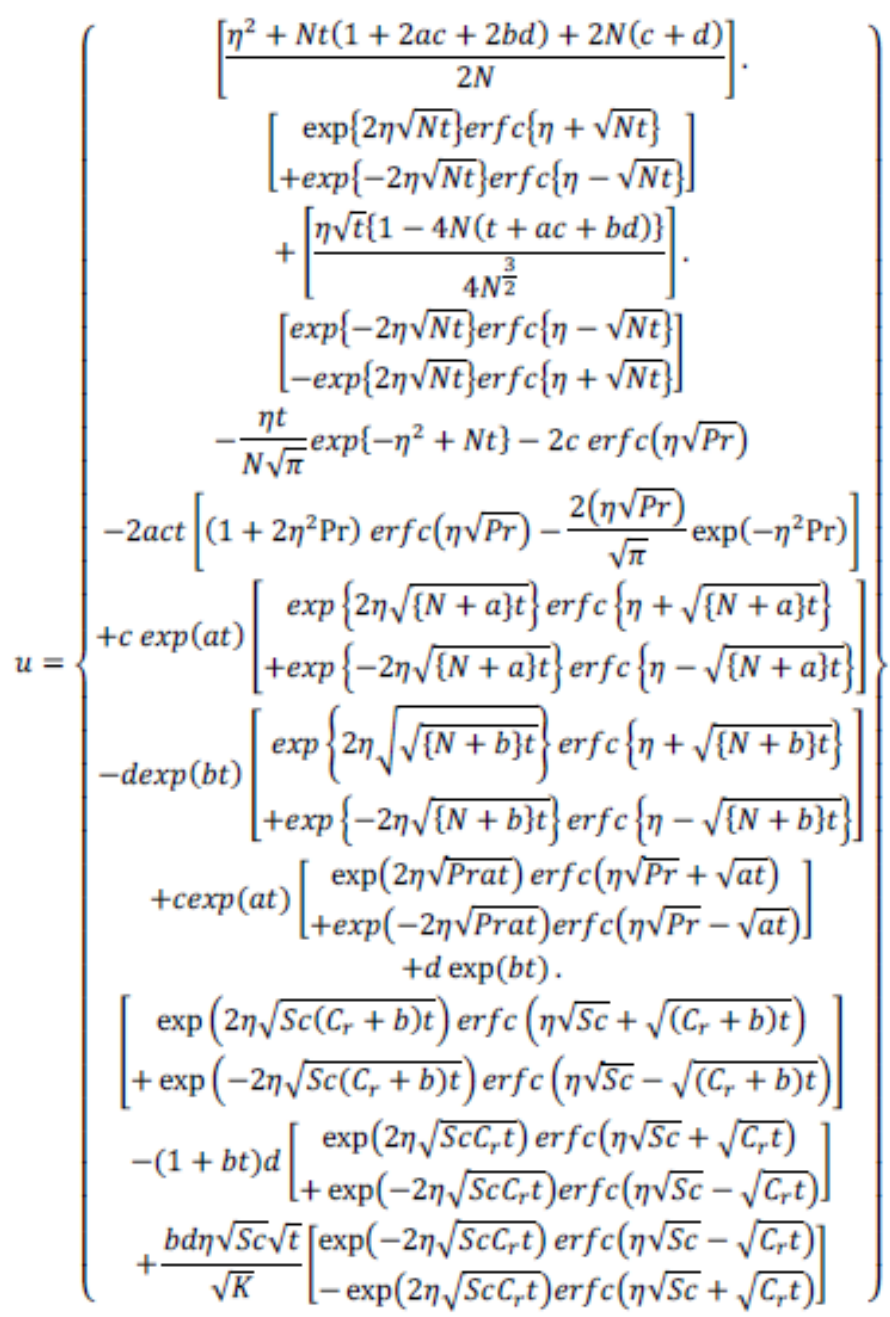

where

$$
\begin{gathered}
N=M+K^{-1}, \quad a=\frac{N}{P r-1}, \quad b=\frac{\left(M+K^{-1}\right)-C_{r} S c}{S c-1}, \\
c=\frac{G r \quad}{2 a^{2}(1-P r)}, \quad d=\frac{G m}{2 b^{2}(1-S c)}, \quad \eta=\frac{y}{2 \sqrt{t}}
\end{gathered}
$$

\section{VALIDITY}

The comparison of the present work with the previous work done by Muthucumaraswamy and Velmurugan (2015) is presented in Table-6.4(a) without porosity of the medium when $G r=G m=5, M=5, t=0.2$, $C_{r}=0.1$ :

\begin{tabular}{|c|c|c|c|c|c|c|}
\hline \multicolumn{5}{|c|}{ Table-I: Velocity field for $P r$} \\
\hline & \multicolumn{5}{|c|}{ Present work } & \multicolumn{3}{c|}{ Muthucumaraswamy and Velmurugan } \\
\hline $\mathrm{Y}$ & $\mathrm{Pr}=0.2$ & $\mathrm{Pr}=0.4$ & $\mathrm{Pr}=0.6$ & $\mathrm{P}=0.2$ & $\mathrm{Pr}_{1}=0.4$ & $\mathrm{P}_{1}=0.6$ \\
\hline 0 & 0.285895 & 0.239544 & 0.207347 & 0.285885 & 0.239538 & 0.207319 \\
\hline 0.2 & 0.225094 & 0.188194 & 0.160509 & 0.225061 & 0.188159 & 0.160511 \\
\hline 0.4 & 0.163767 & 0.135083 & 0.113088 & 0.163753 & 0.135090 & 0.113072 \\
\hline 0.6 & 0.110581 & 0.0888176 & 0.072593 & 0.110577 & 0.088828 & 0.072574 \\
\hline \hline 0.8 & 0.069761 & 0.053565 & 0.042375 & 0.069777 & 0.053565 & 0.042375 \\
\hline 1 & 0.04162 & 0.0297211 & 0.022444 & 0.04154 & 0.029735 & 0.022451 \\
\hline
\end{tabular}


The comparison of the present results with previously published work has established the good agreement and it has been observed that the absolute difference between these comparisons for the flow velocity is less than 10-5. It is seen from the Table-I that the flow velocity are increased with the increasing values of Prandtl number (Pr).

\section{RESULTS AND DISCUSSION:}

In order to get a clear insight of the physical problem, numerical results are displayed with the help of graphs in details. This enables us to carry out the numerical calculations for the distribution of the velocity, temperature and concentration across the boundary layer for various values of the parameters. In the present study we have chosen $M=5, G r=G m=5, t=0.1, K=0.1, S c=0.6$, $P r=0.71$ and $C_{r}=0.1$.

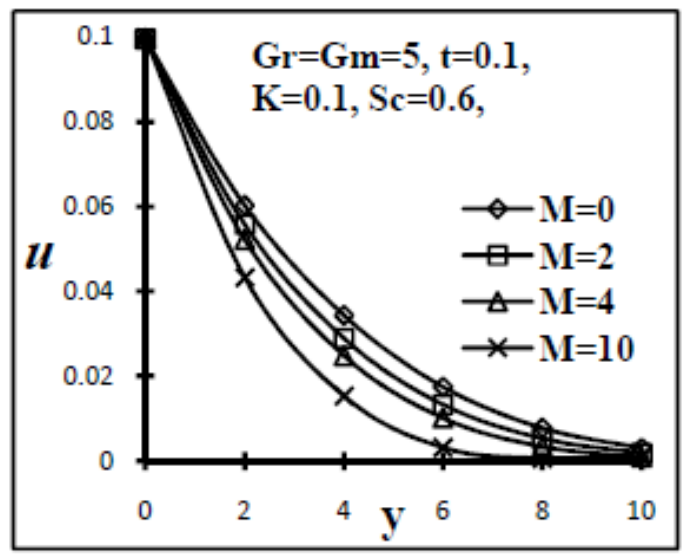

Fig. 2: Velocity profile for magnetic field, $M$

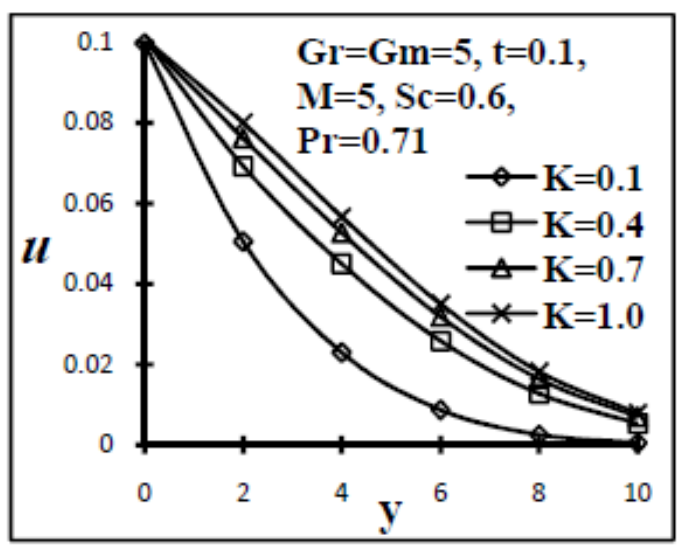

Fig. 3: Velocity profile for porosity, $K$

The effect of the Hartmann number $M$ on the dimensionless velocity profile $\mu$ has been presented in Fig. 2. It is observed in this Figure that the velocity is decreased with the increase of the magnetic parameter. The effects of a transverse magnetic field give rise to a resistive-type force called the Lorentz force. This force has the tendency to slow down the motion of the fluid flow.

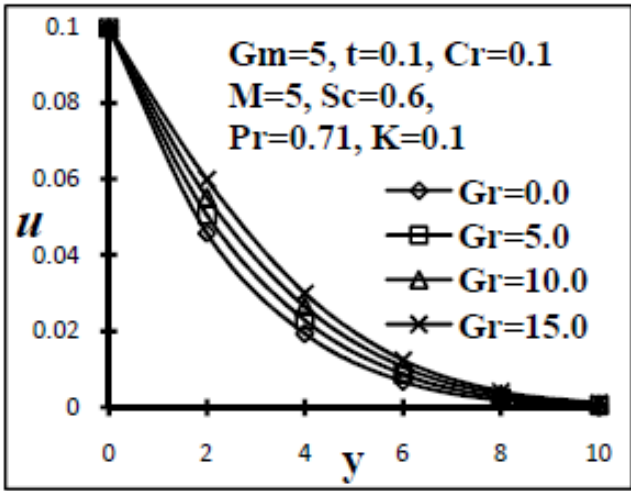

Fig. 4: Velocity profile for free convection parameter, $\mathrm{Gr}$

Figure 3 shows the velocity profiles for different values of the permeability $(K)$. Cleary the increasing $K$ gets the peak value of velocity. These results could be very useful in deciding the applicability of enhanced oil recovery in reservoir engineering.

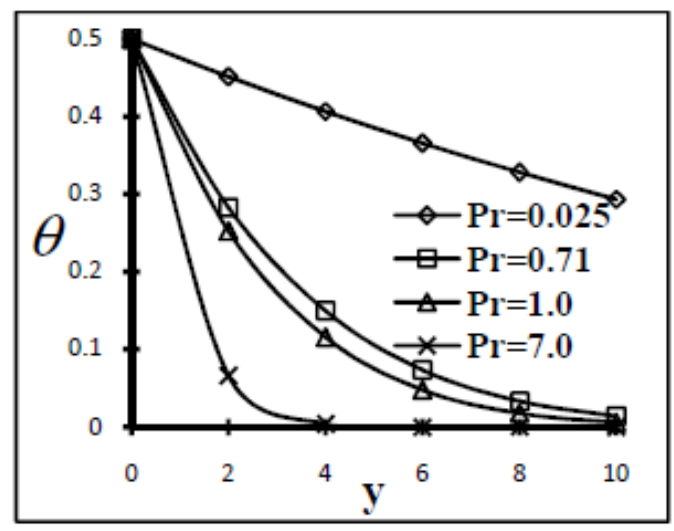

Fig. 5: Temperature profile for Prandtl parameter,

$\mathrm{Pr}$

Figure 4 depicts the effects of the local thermal free convection (Gr) with increasing y on the dimensionless velocity. It is observed that the increase of the Grashof number leads to the increase of the velocity of the fluid. This is because the increase of $\mathrm{Gr}$ results in the increase of temperature gradients $(\mathrm{Tw}-\mathrm{T} \infty)$, leads to the enhancement of the velocity due to the enhanced convection. A typical variation of the temperature profile along the span wise coordinate y is shown in Fig. 5 for different values of Prandtl number (Pr). The results show that an increase of Prandtl number decreases the temperature profiles of thermal boundary layer thickness and more uniform temperature distribution across the boundary layer. Moreover, the velocity becomes linear due to the metallic liquid $(\operatorname{Pr}=0.25)$. 


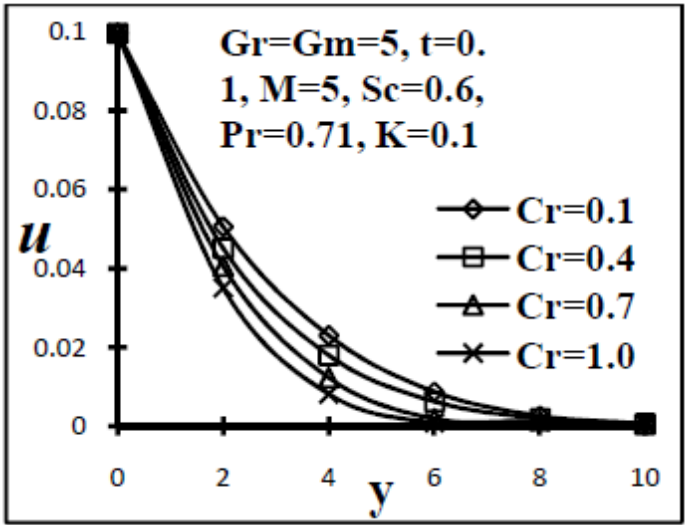

Fig. 6: Velocity profile for chemical reaction, $C_{r}$

The effects of the generative chemical reaction parameter $\mathrm{Cr}$ on the dimensionless velocity $\mu$ and concentration $\phi_{i}$ profiles are respectively presented in Figs 6 , and 7 . It is observed in these Figures that the velocities as well as concentration are decreased with the increase of the generative chemical reaction. The effect of chemical reaction parameter is very important in the concentration field. Chemical reaction increases the rate of interfacial mass transfer. Reaction reduces the local concentration, thus increases its concentration gradient and its flux.

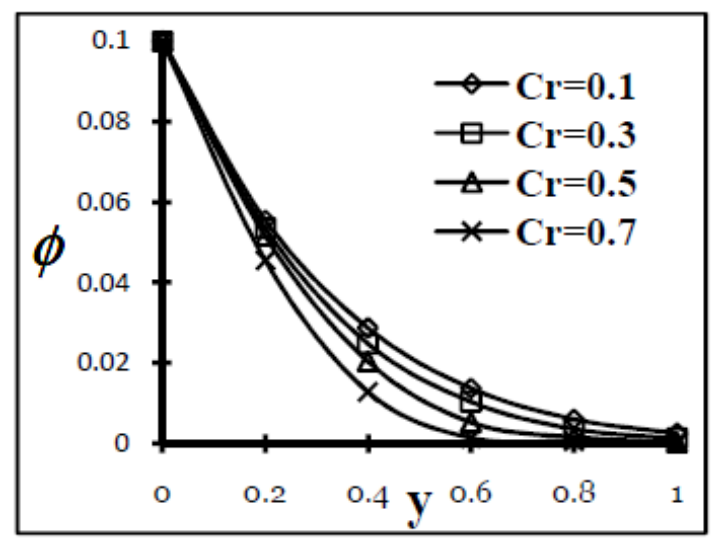

Fig. 7: Concentration profile for chemical reaction, $C_{r}$

Figure 8 illustrates the concentration profiles across the boundary layer for various values of Schmidt number Sc. The figure shows that an increasing in Sc results in a decreasing the concentration distribution, because the smaller values of $\mathrm{Sc}$ are equivalent to increasing the chemical molecular diffusivity.

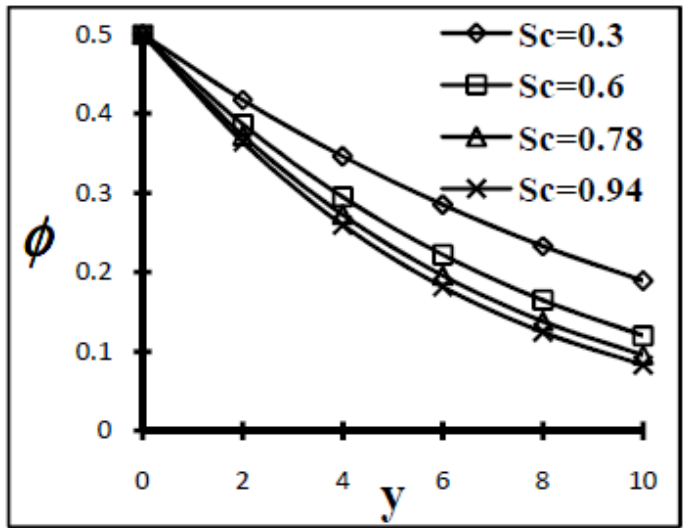

Fig. 8: Concentration profile for Schmidt number, Sc

\section{CONCLUSIONS}

In this paper we have studied analytically the porosity and chemical reaction effects on an unsteady MHD free convention and mass transfer flow past a semi-infinite vertical plate embedded in a Darcian porous medium. From the present study the following conclusions can be drawn:

- An increase in magnetic field parameter decreases the velocity profiles.

- The velocity profiles are increased with an increase of free convection currents and porosity of the medium as well.

- The generative chemical reaction of the species leads to decrease of the flow velocity as well as concentration

- boundary layer.

- Large Darcy number (large porosity of the medium) leads to the increase of the velocity of the fluid within the boundary layer.

- The Prandtl number for the temperature field leads to decrease the thermal boundary layer.

- The concentration boundary layer has been reduced for the effect of Schmidt number

\section{REFERENCES}

[1]. K.D. Dorfman, and H. Brenner, Modeling DNA electrophoresis in microfluidic entropic trapping devices, Biomedical Microdevices, 4(3), 2002, 237-244.

[2]. J.M.P.Q. Delgado, Mass transfer from a plane surface immersed in a porous medium with a moving fluid, Chem. Eng. Res. Des. 85, 200, 386-394.

[3]. L. Minkin, Thermal diffusion of radiation in porous media, Ra-diat. Prot. Dosim., 106, 2003, 267-272.

[4]. T. Seo, H.-D. Kim, J.-H. Choi, and J.H. Chung, Mathematicalmodeling of flow field in ceramic candle filter, J. Therm. Sci. , 7, 1998, 85-88. 
[5]. H.B. Al-Saffar, B. Ozturk, and R. Hughes, A Comparison of porous and non-porous gasliquid membrane contactors for gas separation, Chem. Eng. Res. Des., 75, 1997, 685-692.

[6]. A.J. Chamkha, and A.R.A. Khaled, Hydromagnetic combined heat and mass transfer by natural convection from a permeable surface embedded in a fluid satural porous medium, Int. J. Numerical Methods for Heat and Fluid Flow, 10(5), 2000, 455-476.

[7]. B. Mukherjee, and N. Prasad, Effect of radiation and porosity parameter on hydromagnetic flow due to exponentially stretching sheet in a porous media, Int. J. Engineering, Science and Technology, 6(1), 2014, 58-70.

[8]. S. Ahmed, Free and Forced Convective MHD Oscillatory Flows over an Infinite Porous Surface in an Oscillating Free Stream, Latin American Applied Research, 40, 2010, 167- 173.

[9]. J.G. Kumar, and R.M. Ramana, Viscous dissipation effects on MHD flow past a parabolic started vertical plate with variable temperature and mass diffusion, Int. J. Scientific \& Engineering Research, 6(9), $2015,57$.

[10]. R. Muthucumaraswamy, and A.N. Armstrong, Mass transfer effects on flow past a parabolic started vertical plate with variable temperature and mass diffusion, Int. J. Mathematical Archive, 5(2), 2014, 53-58.

[11]. M. Muralidharan, and R. Muthucumaraswamy, Parabolic started flow past an infinite vertical plate with uniform heat flux and variable mass diffusion, Int. Journal of Math. Analysis, 8(26), 2014, 1265 -1274 .

[12]. R. Muthucumaraswamy, and S. Velmurugan, Theoretical study of heat transfer effects on flow past a parabolic started vertical plate in the presence of chemical reaction of first order, Int. J. Applied Mechanics and Engineering, 19(2), 2014.

[13]. R. Muthucumaraswamy, and J. Venkatesan, Radiative flow past a parabolic started isothermal vertical plate with uniform mass flux, Int. Journal of Math. Analysis, 7(59), 2013, 2907-2921.

[14]. A. Raptis, G. Tzivanidis, and N. Kafousias, Free convection and mass transfer flow through a porous medium bounded by an infinite vertical limiting surface with constant suction, Letters Heat Mass Transfer, 8, 1981, 417-424.
[15]. A. Raptis, N. Kafousias, and C. Massalas, Free convection and mass transfer flow through a porous medium bounded by an infinite vertical porous plate with constant heat flux, ZAMM, 62, 1982, 489-491.

[16]. A. Raptis, and C. Peridikis, Radiation and free convection flow past a moving plate, Int J. Applied Mechanics \& Engineering, 4(4), 1989, 817-821.

[17]. S. Ahmed, Modelling of rotational fluid with an accelerated vertical plate embedded in a Darcian porous regime, Int. J. Engineering, 11(3), 2013, 69- 76.

[18]. Chaudhary, R. C. and Jain, A. Magnetohydrodynamic transient convection flow past a vertical surface embedded in a porous medium with oscillating temperature, Turkish Journal of Engineering and Environmental Sciences, Vol. 32, No. 1, pp. 13-22 (2008).

[19]. K. Hamida, and S. Ahmed, Free Convective Heat Transport in a Porous Media bounded by an isothermal vertical plate with thermal Radiation and Magnetohydrodynamic effects: An Exact Solution, IOSR Journal of Applied Physics, 7(3), 2015, 9-17.

[20]. A.K. Agrawal, N.K. Samria, and S.N. Gupta, Free convection due to thermal and mass diffusion in laminar flow of an accelerated infinite vertical plate in the presence of magnetic field, Journal of Heat and mass transfer, 20, 1998, 35-43.

[21]. A.K. Agrawal, N.K. Samria, and S.N. Gupta, Study of heat and mass transfer past a parabolic started infinite vertical plate, Journal of, Heat and mass transfer, 21, 1999, 67-75.

[22]. R. Muthucumaraswamy, and S. Velmurugan, Chemical reaction effects on flow past a parabolic started Vertical plate with variable temperature and mass diffusion in the presence of magnetic field, Int. J Engineering, 8(2), 2015, 175-179.

[23]. K. Manivannan, R. Muthucumarswamy, and V. Thangaraj, Radiation and chemical reaction effects on isothermal vertical oscillating plate with variable mass diffusion, Thermal science, 13(2), 2009, $155-162$.

[24]. S. Ahmed, and K. Kalita, MHD transient flow through a porous medium bounded by a hot vertical plate in presence of radiation: a theoretical analysis, J Eng Phys Thermophysics, 86(1), 2013, 31-39.

[25]. S. Ahmed, Numerical analysis for MHD chemically reacting and radiating fluid past a non-isothermal impulsively started vertical 
surface adjacent to a porous regime, Ain Shams Eng J., 5, 2014, 923- 933.

[26]. S. Ahmed, J. Zueco, and L.M. LópezGonzález, Effects of chemical reaction, heat and mass transfer and viscous dissipation over a MHD flow in a vertical porous wall using perturbation method, Int. J. Heat and Mass Transfer, 104, 2017, 409-418. 